\title{
Trabajo social y castigo corporal: el significado atribuido por profesionales de los servicios sociales de atención a la infancia en España
}

\author{
Social work and corporal punishment: the meaning attributed by \\ professionals of social services of childcare in Spain
}

\author{
Picornell-Lucas, Antonia, ${ }^{1 *}$ Ph.D, Rivera Álvarez, Joaquín, ${ }^{2}$ Ph.D. \\ ${ }^{1}$ Universidad de Salamanca (USAL) (España). Fundadora y Presidenta de REDidi; Instituto de Iberoamérica \\ (USAL). ${ }^{2}$ Universidad Complutense de Madrid (UCM) (España). Correspondencia: toi@usal.es
}

Recibido: 14-07-2016; Aceptado: 31-10-2016

\section{RESUMEN}

Este trabajo presenta los resultados de una investigación centrada en analizar las opiniones de los profesionales de atención a la infancia con respecto al castigo corporal. Trece trabajadores sociales de la Comunidad de Madrid han sido entrevistados para determinar sus percepciones sobre el uso del castigo corporal como estrategia educativa familiar. Para explorar sus actitudes y sentidos se utiliza el grupo de discusión, dentro del enfoque cualitativo. Los resultados muestran actitudes contradictorias entre los participantes. Se defiende la importancia de erradicar el castigo corporal, aunque se manifiesta a la vez que su empleo no tiene consecuencias negativas. Otros temas debatidos y analizados han sido: factores estresantes, transmisión intergeneracional, prácticas de crianza, eficacia atribuida y prohibición legal. De todo ello se puede concluir que el uso del castigo corporal como método de crianza no causa impresión negativa en los trabajadores sociales, apuntando a la importancia de la cultura en el mantenimiento de tales actitudes.

Palabras clave: Infancia, castigo corporal, actitudes disciplinarias, trabajadores sociales, política social. 
Picornell y Rivera - Trabajo social y castigo corporal: atención a la infancia en España

\section{ABSTRACT}

This work presents the results of a research focused on analyzing the opinions of childcare professionals with regard to corporal punishment. Thirteen social workers of the Community of Madrid have been interviewed to determine their perceptions on the use of corporal punishment as a family educational strategy. To explore their attitudes and ways the focus group is used, within the qualitative approach. Results show contradictory attitudes among participants. The importance of removing corporal punishment is supported, although it is also stated that its use has no negative consequences. Other topics discussed and analysed were: stressful factors, intergenerational transmission, parenting practices, effectiveness and legal prohibition. From all this we can conclude that the use of corporal punishment as a parental practice does not produce negative impression on social workers, pointing the importance of culture in maintaining such attitudes.

Keywords: Childhood, corporal punishment, discipline, social workers, social policy.

\section{INTRODUCCIÓN}

El número de niños víctimas de violencia en el mundo no se ha reducido, a pesar de los esfuerzos de las naciones (Hart, Lee y Wernham, 2011). Y una de esas formas de violencia, "más común en el mundo industrializado" (UNICEF, 2003: 23), es el castigo corporal, confirmándose la frecuencia de su uso (Lansford y otros, 2010; Straus, 2010; Tang, 2006).

No se trata de un problema nuevo, aunque habitualmente suele asimilarse a la disciplina correctiva, pero esta última incluye también conductas parentales positivas (Straus y Fauchier, 2011). Este ejercicio positivo de la parentalidad no implica permisividad, sino el respeto de los derechos de los niños y una educación sin violencia, poniendo los límites necesarios. A este respecto, el Comité de Derechos del Niño, en su Observación General no. 8 (2006), exige "la eliminación de toda disposición que permita cierto grado de violencia contra los niños en sus hogares o familias o en cualquier entorno" (párrafo 31), en consonancia con la Convención de los Derechos del Niño (CDN) (1989), que defiende la protección a la infancia de "todas las formas de violencia física o mental, abuso, negligencia, maltrato o explotación" (art. 19).
Este tipo de comportamiento no debe confundirse con el maltrato infantil; son actitudes diferentes (Mahmoud, Salah-El-Din e Ibrahim, 1998; Whipple y Richey, 1997). El maltrato infantil supone una anomalía en las interacciones sociofamiliares, con una intención premeditada de dañar la integridad física y psicológica del niño, mientras que la motivación del castigo corporal es la corrección disciplinaria, la cual puede incluir tanto conductas positivas como negativas (Straus y Fauchier, 2011). El castigo corporal es el uso de la fuerza física y/o la agresión psicológica degradante para infligir dolor, pero no injurias, con el fin de disciplinar al hijo o controlar su mala conducta (Straus y otros, 1998; Straus y Fauchier, 2011), mediante bofetadas, puntapiés, mordiscos, tirones de pelo, humillaciones, insultos, amenazas, etc.

La dificultad en diferenciar ambas conductas está ocasionando una confusión en los padres y madres, a la vez que condiciona la prohibición legal del uso del castigo corporal en la familia; tal y como se hizo en la escuela en el año 1985, al prohibirse cualquier tipo de castigo físico y/o psicológico por parte del profesorado. La modificación del art. 154 del Código Civil español, en el año 2007, señala que la patria potestad ha de ejercerse en beneficio 
de la integridad física y psicológica del menor. Y la L.O. 8/15 de 22 de julio, de modificación del sistema de protección a la infancia y a la adolescencia incluye como criterio general a ponderar por todo responsable público: "La conveniencia de que su vida y desarrollo tenga lugar en un entorno familiar adecuado y libre de violencia" (art. 3.1.c). Pero al no existir una ley específica que prohíba el castigo corporal, "los remedios que el ordenamiento fija para el castigo corporal no son creadores de cultura y estarán condicionados por las representaciones sociales que cada funcionario de la administración de los servicios sociales o de la justicia tenga sobre el castigo como medio educativo" (Picornell-Lucas y otros, 2015: 119).

En efecto, la aceptación social del uso del castigo corporal es una de las realidades que inducen a su utilización, según las investigaciones epidemiológicas consultadas (Belsky, 1993; Holden, Coleman y Schmidt, 1995; Jackson y otros, 1999). Pues "así como las funciones naturales vienen garantizadas por la herencia biológica, las superiores o culturales se desarrollan sobre las arquitecturas socioculturales históricas que ofrece el medio humano" (del Río y Álvarez, 1997: 104), por lo que un predictor del castigo corporal sería la internalización de tales creencias en normas y valores. Ello supone que tanto las manifestaciones lingüísticas como las conductas parentales son aprendidas por identificación y apropiadas desde la sociedad. $Y$, pese a no ser la única manera para corregir la mala conducta, ya que, en ocasiones, no la minimiza sino que aumenta su frecuencia (Lansford, 2010), su uso y defensa están extendidos a nivel mundial, por tradiciones culturales 0 argumentos religiosos, con independencia de su eficacia y consecuencias: problemas de ansiedad, deterioro cognitivo, conductas antisociales, entre otras (Berlin y otros, 2009; Gershoff, 2002; Pinheiro, 2006; Russell y Russell, 1996; Straus, 1991, 1994, 2010; Taylor y otros, 2010).
Como se evidencia en el estudio del CIS (2005), más de la mitad de los encuestados españoles cree necesario y positivo pegar a un niño para modificar la conducta no deseada. Idénticos resultados son los alcanzados por Bussmann (2009) en un estudio comparativo entre cinco países europeos - Suecia, Austria, Alemania, Francia y España-, destacando Francia $(71,5 \%)$ y España (54.6\%) con una mayor proporción de padres que aceptan y utilizan el castigo corporal, justificado en las creencias tradicionales y en la falta de alternativas. Por otra parte, el reciente informe mundial desarrollado por Global Initiative to End all Corporal Punishment of Children (2016) revela, entre otros datos, que el $81 \%$ de los padres en EEUU, el $85 \%$ en China, el $70 \%$ en Argelia, el $76 \%$ en Pakistán o el $43,1 \%$ en Guatemala cree necesario el uso del castigo corporal ante la desobediencia de los hijos.

Se están realizando estudios que intentan establecer una causalidad, asignando condiciones como el género, el estatus socioeconómico, la predisposición genética, la creencia en su efectividad (Gershoff y Bitensky, 2007) o la prohibición legal (Bussmann, 2009). Otros marcos explicativos atribuyen la etiología del castigo corporal a un origen multidimensional e infieren los motivos de su defensa y uso como resultado de la interacción de fuerzas sociales estresantes para los padres y madres. Aquí se encuentran el modelo ecosistémico (Belsky, 1993), que describe la interrelación entre el microsistema familiar, el entorno más inmediato de organización de la comunidad y la sociedad con sus normas culturales y legislación específica, o el modelo sociointeraccional (Wolfe, 1985), que defiende la influencia de variables individuales y sociales en las conductas educativas parentales.

A pesar de la necesidad de profundizar en las opiniones de la población ante el castigo corporal y en su uso como 
método disciplinario, imprescindible para garantizar una protección infantil completa ante la violencia, en España siguen siendo escasos los estudios que ofrecen datos actuales sobre las actitudes a favor de su uso, entre ellos los de Calvete, Gámez-Guadix y Orue (2010), Gracia y Herrero (2008) o Juste, Morales y Costa (1997). Y esta falta de respuestas y políticas públicas de intervención facilita que la sociedad conserve actitudes favorables hacia el castigo corporal. Para visibilizar el problema, el objetivo de este estudio es explorar el significado atribuido y los valores que sobre el uso del castigo corporal tiene una muestra de trabajadores sociales madrileños del campo de la Protección Social a la infancia, y describir los factores que intervienen en la legitimación de tal conducta.

\section{METODOLOGÍA}

Desde la lógica cualitativa de los grupos de discusión, rápidos y flexibles, que permiten integrar objetivos de investigación para entender las realidades sociales (Llopis, 2004), se definió la muestra en función de los siguientes atributos: zona geográfica: Comunidad Autónoma de Madrid; profesionales de las instituciones del Sistema de Servicios Sociales (público y privado); campo de trabajo: infancia; trabajadores y trabajadoras sociales en ejercicio; no haber participado nunca en un grupo de discusión. Se atendió también a las características principales de la técnica del grupo de discusión: homogeneidad, sin que mediara conocimiento entre los participantes para no degradar la dinámica del grupo, y heterogeneidad en cuanto al ámbito geográfico de actuación profesional (rural, semiurbano y urbano; localidades grandes y pequeñas).
El universo quedó constituido por 11 trabajadores sociales (10 mujeres y 1 varón) del Sistema de Servicios Sociales de la Comunidad Autónoma de Madrid. Provenían de: el Centro de Ejecución de Medidas Judiciales 'José de las Heras'; la Comisión de Tutela del Menor (Instituto Madrileño del Menor y de la Familia); el Equipo de Orientación Educativa de Moratalaz; Equipos de Familia de Leganés, Fuenlabrada, Rivas Vacía Madrid y MISECAM-Mancomunidad Este-Villarejo de Salvanés; el Centro de Atención a la Infancia del ayuntamiento de Madrid; Save the Children; la Fundación ANAR y un equipo autónomo.

La muestra se dividió en dos grupos heterogéneos como estrategia para una posterior evaluación paralela de sus cogniciones sociales. La descripción de los grupos se muestra en la figura 1. Posteriormente, analizados los constructos internos de cada grupo, los informes individuales de cada uno de ellos presentaron perspectivas similares con relación al objeto de estudio.

De los discursos originados en ambos grupos, y eliminada la información irrelevante, se identificaron unas unidades textuales genéricas de registro - palabra, tema- (Krippendorff, 1990), atendiendo tanto a su frecuencia de aparición como a su significado; y se codificaron en categorías y subcategorías de análisis (Figura 2) para una mejor comprensión. La construcción de categorías tomó como punto de partida los principios de exclusión mutua, homogeneidad y que fueran pertinentes al objetivo de la investigación (Bardin, 1986), esto es, reveladores de los valores, creencias y actitudes de los participantes con relación al castigo corporal. La interpretación del sentido del discurso se realizó con el desarrollo de matrices a través de las cuales se descubrieron las relaciones entre las categorías. 
Revista Búsqueda - Julio/Diciembre de 2016

Figura 1. Descripción de los grupos de discusión: GD1 y GD2

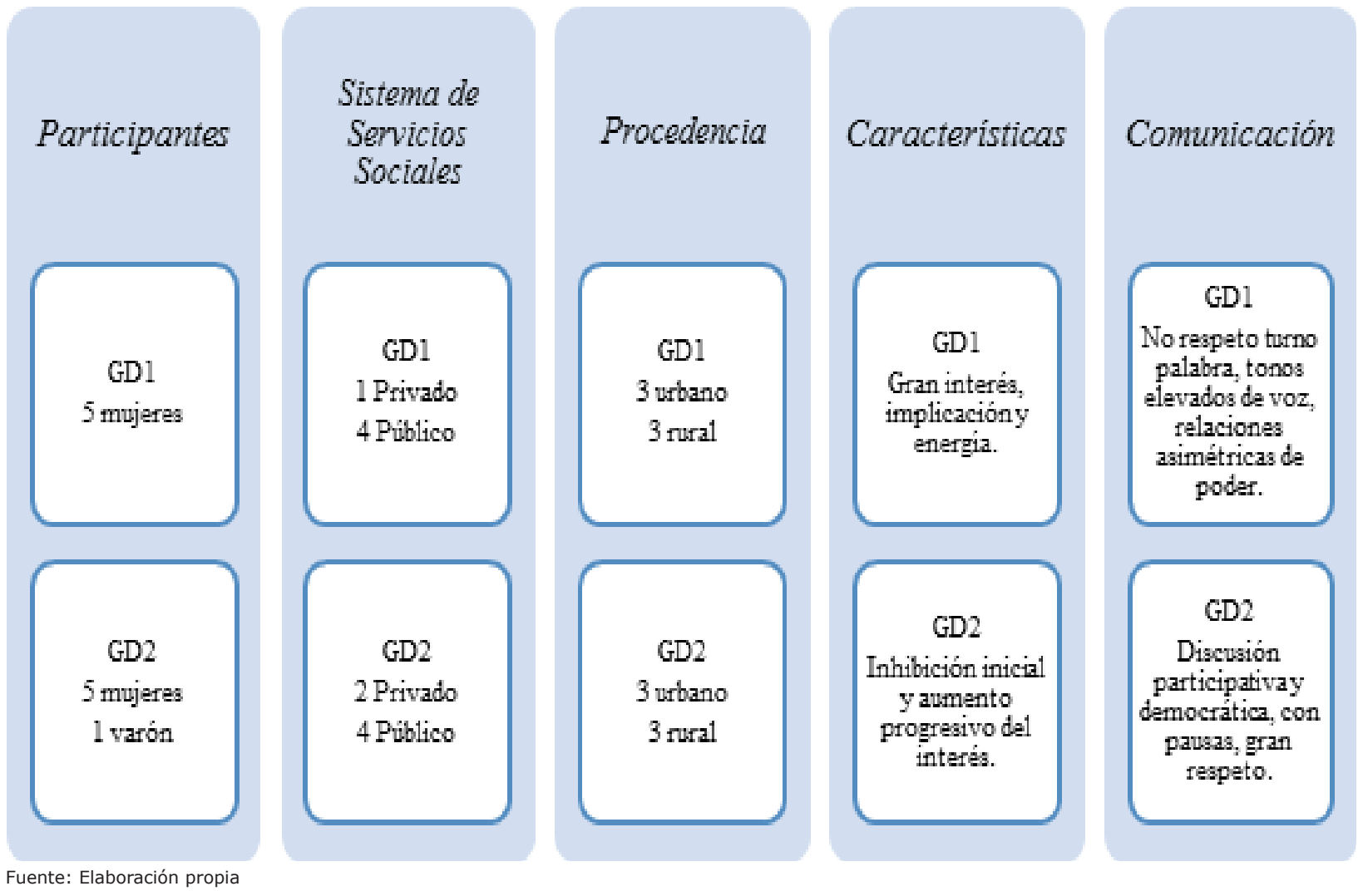

Figura 2. Codificación de las unidades textuales en categorías

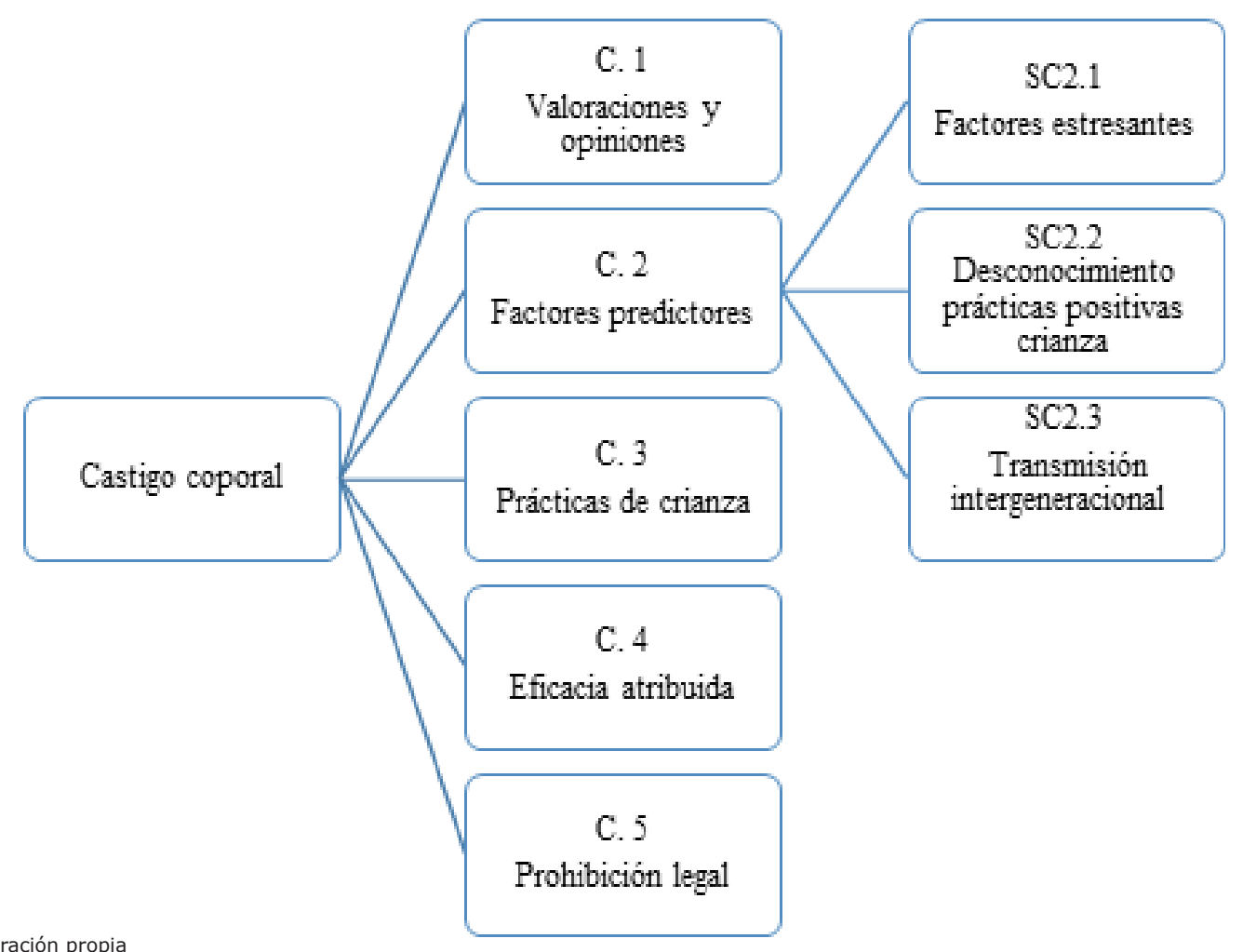


Picornell y Rivera - Trabajo social y castigo corporal: atención a la infancia en España

\section{RESULTADOS}

La percepción del castigo corporal en ambos grupos es semejante y quedaron manifiestas dificultades para diferenciar el castigo corporal del maltrato: "Si se acepta eso, es aceptar el maltrato físico" (GD2; 109); "¿El cachete habitual sería maltrato?" (GD2, líneas 502-503). Y aunque dicho conflicto no se resuelve a lo largo de la discusión, sí existe un conocimiento sobre el significado del castigo corporal sobre el que se construye todo el discurso. "Porque el castigo físico no es solamente pegar... o sacarle a la terraza con el frío... Pero es constantemente voces y voces y voces" (GD1; 384-387).

En la primera de las categorías: C1. Valoraciones y opiniones sobre el castigo corporal se defendieron valoraciones contrapuestas. Los trabajadores sociales se posicionan en contra del uso de la violencia como método educativo, por la degradación que representa: "A mí lo que me parece peor del castigo físico es la humillación animal que supone, el abuso de poder del fuerte contra el débil" (GD2; 826-828); "yo no veo que nunca sea tiempo de un cachete, me parece que siempre tiene que haber otras alternativas" (GD1; 343-344). Aunque, paralelamente admiten que no entraña peligro para el niño: "Si se le da con la mano es muy puntual, muy excepcional, y en un hecho concreto. Estar dándole en la mano es como si le doy en la cara, pero sí es verdad que dar una bofetada, para mí, tiene una magnitud y un daño, también mayor que si se da en un momento determinado para quitar de un peligro concreto. Insisto, siempre tiene que ir referido desde lo educativo a ese momento concreto" (GD2; 237-244).

En los factores predictores relacionados con el castigo corporal (C2), se manifestaron tres subcategorías: Factores estresantes, desconocimiento de los padres sobre prácticas positivas y transmisión intergeneracional.
SC2.1: Los factores estresantes son la primera causa para justificar el castigo corporal. En su discurso, surgen razones como el estrés, el número de hijos, la monoparentalidad y el bajo nivel educativo, entre otros. "Pienso que los padres educan... de la mejor forma posible y que en algunas ocasiones la falta de habilidades personales, o las impotencias, situación económica, la situación de estrés,..." (GD2, líneas 140142). "La violencia la tenemos muchísimo más incorporada, desde todo ese estrés, desde la poca tolerancia que tenemos, desde que no aguantamos al otro" (GD2; 1351-1352).

SC2.2: Un segundo factor que se percibe también como condicionante para el uso del castigo corporal, según los participantes, es la falta de formación.

"Creo que responde a una carencia de habilidades educativas..." (GD1, líneas 309-310). Se defienden determinadas actitudes justificando que no se conocen otras alternativas: "Cuando un niño... comete un error, lo normal es que comentamos errores contantemente, porque no sabemos que se hace de otra manera" (GD2; 544-547); "No somos capaces... por no entender o no tener capacidad o autoridad... en un momento determinado" (GD2; 274-276). El discurso construido apunta al desconocimiento parental sobre los sentimientos y pensamientos de los niños y la necesidad de fortalecerles en su tarea educativa: "El tema es dar estrategias para que eso se pueda controlar" (GD1; 594-595).

SC2.3: Por otra parte, los participantes plantean el castigo corporal como modelo de aprendizaje, "...de una historia personal, de lo que ha vivido o de la cultura" (GD1; 517-518); "hay mucha gente que tiene un estrés increíble, no ha tenido buenos modelos de referencia y lo incorpora como medio habitual de educación a sus hijos" (GD2; 615-617); "en el programa ves cómo normalizan y justifican la violencia que ellos han vivido y la emplean con sus hijos" (GD1; 401-402). 
Se manifiesta que la violencia es un rasgo cultural, transmitida entre generaciones, de padres a hijos, por medio de unos patrones: "Hay personas que utilizan el castigo físico de forma habitual y los niños están acostumbrados a eso, incluso han crecido y no tienen conciencia de que sea nada raro, sino que lo introducen como un modelo de aprender" (GD2; 63-65).

Las Prácticas de crianza (C3) es otra categoría destacada en el discurso. Los participantes defienden que "todos quieren educar de la mejor manera a sus hijos" (GD2; 9-10); "otra cosa son las técnicas que utilizan para ello... y cómo se manejan, con los límites, autoridad y castigos..." (GD2; 13-14). Se reflexiona sobre las estrategias parentales adecuadas para conseguir una conducta apropiada en el niño. Ambos grupos destacan el cambio en los estilos educativos, evolucionando desde el autoritarismo a la permisividad, obviando la disciplina inductiva o de razonamiento desde la cual se educa en la autonomía y cooperación, obteniendo el niño mayores competencias. Manifiestan: "El caso es que a lo mejor le has dado de más y no lo que él necesita, que es atención" (GD2; 1049-1050); o "los niños están súper-protegidos y súpercubiertos materialmente de todo y súperconsentidos, y... eso es lo que ha hecho el despunte de esta agresividad" (GD1; 431-433). No obstante, se afirma que "da igual, niños a nivel económico muy carentes, niños a nivel económico con absolutamente todo, es que en los dos coincide lo mismo, falta de límites y falta de referentes" (GD1; 439-442); "Sigue habiendo una incapacidad en los padres en imponer normas" (GD 2, línea 91).

Se defiende que la educación de los padres y madres ha de "mantener una línea coherente, que el niño pueda identificar con el paso del tiempo qué es lo que se admite o no se admite, qué es lo que está bien o lo que está mal, con quién sí y con quién no. O sea, la constancia, las rutinas" (GD1; 1143-1145). Pero, a la vez, manifiestan una actitud positiva hacia el castigo corporal, en caso necesario, alegando su utilización en ocasiones: "Yo por lo menos lo he hecho (carcajada reafirmando su discurso) y no lo entiendo un maltrato. Lo entiendo como una forma de limitar, porque hay un riesgo, hay un peligro y entonces doy" (GD2, líneas 193195). El grupo mantiene un discurso con afirmaciones confusas, pensamientos contradictorios.

En cuanto a la consideración de la Eficacia atribuida (C4) al castigo corporal, los trabajadores sociales defienden la utilidad del castigo corporal: "te tiene que ayudar a ser mejor... pero si te va a dañar deja de ser efectivo" (GD2; 855-857); "Sé que mi hijo deja de hacer esto, de tocar este vaso porque me está poniendo muy nerviosa. Si le pego un bofetón lo va a dejar de tocar porque es inmediato, es causaefecto. Entonces, le doy el bofetón" (GD1; 1048-1050). La validez del uso del castigo corporal que con más frecuencia exponen los participantes es la que hace referencia a la agresividad y al uso de la violencia para la resolución de un problema: "Si hablamos de adolescentes hemos perdido todo el control y toda la capacidad de negociación... en el momento en el que le ponemos la mano encima" (GD2; 220222); "El niño efectivamente aprende a crecer con una cierta violencia. ... si yo quiero que el otro haga algo, que me escuche, que me haga caso, es a través de la violencia" (GD2; 233-235).

Aunque no ha habido una manifestación explícita a favor de la Prohibición legal (C5) del castigo corporal, se deduce la necesidad del grupo de conferirle un status legal. Se afirma que "las leyes tienen que ser inequívocas, o sea, no claras, inequívocas" (GD1; 502). Esto indica la necesidad de una prohibición expresa del castigo corporal, que ayudaría a hacerlo visible y a establecer un procedimiento administrativo para su denuncia y posterior intervención; aspecto también reflejado en sus palabras: "según la ley es que todo adulto conocedor de... debe ponerlo en manos de los profesionales adecuados" (GD1; 1078-1079) o "esas situaciones no llegan porque no está incorporado 
como que eso sea algo muy inadecuado" (GD2; 971-972). "La sociedad española se ha dotado de unas leyes que protegen precisamente a la infancia y que por eso no es que solamente nos hayamos dotado de esas leyes, sino que además hemos tenido que buscar alternativas a esa estrategia educativa, entonces, en ese sentido hemos ido avanzando" (GD1; 130-133), alusión que hace referencia al avance que supone la legislación en la protección de los derechos de la infancia.

La información ofrecida también ha sido analizada desde la utilización de figuras retóricas que, según Murilloy Mena (2006), son una parte densa del lenguaje, pero también una buena fuente de información que nos puede ayudar a construir el mapa de significados del objeto de estudio. Fue significativa la utilización de metonimias, metáforas y paradojas para explicar las opiniones que los participantes tenían sobre el tema. Algunos ejemplos son:

"Los latinoamericanos y los de los países africanos, por ejemplo etíopes, te dicen claramente, es que ustedes no pegan a los niños" (GD1; 183-185); "...y para ti el manotazo no pasa nada; y a los sudamericanos que están acostumbrados como norma de castigo a darles dos con el cinturón" (GD2, líneas 221-222). Prejuicio sobre una mayor cotidianeidad y severidad en otras culturas.

"...durante el día tenemos estímulos de trabajo negativos... llegamos cargados. $Y$ claro, los niños están ahí reclamando su atención y muchas veces la reclaman mal y nos ponen al límite" (GD2, líneas 585588). De nuevo una parte de la realidad representa al universo.

"...y de ahí ya al maltrato que ya es otro extremo" (GD2, líneas 45-46). Analogía entre castigo corporal y maltrato.

"(Un cachete) es como desahogo" (GD2, línea 609). Metáfora abstracta que asigna significado al castigo corporal.
"Como hecho puntual de suelta eso que te vas a hacer algo (acompaña el discurso con el gesto de darse a sí mismo un manotazo), yo lo compartiría si luego eso va acompañado de un vamos a hablar de no puedes coger esto, porque te va a pasar esto y esto" (GD2, líneas 258260). Contradicción y defensa del castigo corporal como técnica correctiva.

"El que castiga físicamente también piensa que lo hace porque lo quiere" (GD2, línea 810); "Quien más te quiere es el que te agrede, es lo que se decía antes" (GD1; 485-486). Paradojas.

\section{CONCLUSIONES}

Es difícil obtener datos sobre el castigo corporal intrafamiliar (Pinheiro, 2006). Y, aunque la información no debiera restringirse a la incidencia y prevalencia, sino también al desarrollo positivo del niño, es prioritario conocer tales referencias para poder promocionar formas positivas de disciplina; ya que "los recursos públicos son más accesibles cuando los problemas sociales son vistos desde sus consecuencias" (Schmid y Benbenishty, 2011: 1182).

En este estudio se aporta una nueva evidencia sobre las representaciones que los trabajadores y las trabajadoras sociales atribuyen al uso del castigo corporal como metodología educativa parental, en lo relativo al discurso y a la práctica, profundizando en el conocimiento de las causas, la opinión sobre la eficacia atribuida y la actitud ante su prohibición legal. La interacción de los participantes de ambos grupos de discusión ha permitido conseguir el objetivo de comprobar las percepciones y valoraciones que ayudan a legitimar socialmente el uso del castigo corporal. Los resultados son de interés por varias razones: por el aumento de conocimiento sobre el tema; por su influencia en el perfeccionamiento del Sistema de Protección Social a la Infancia; y por el aumento de la sensibilización 
social, en especial de los trabajadores sociales de la red de infancia.

El significado que le atribuyen los trabajadores sociales al castigo corporal difiere entre ellos en relación con su aceptación como técnica disciplinaria. No obstante, aun en el caso de aquellos que defienden su no utilización, lo consideran, en ocasiones, necesario, señalando algunos factores desencadenantes como el estrés, el desconocimiento de prácticas disciplinarias alternativas y la transmisión intergeneracional, argumentaciones coincidentes con otros estudios que manifiestan el incremento en el uso de la disciplina punitiva ante situaciones similares (Dietz, 2000; MacKenzie y otros, 2011; Simons y Wurtele, 2010); e incluso utilizándolo con sus hijos, algunos de ellos, esporádicamente. Sus relatos se acomodan a la teoría del aprendizaje social, argumentado también por otros estudios sobre el castigo corporal (Muller, Hunter y Stollak, 1995; Simons y Wurtele, 2010; Wareham, Paquette y Chavez, 2009). Factores etiológicos todos ellos que debieran ser considerados por las políticas de infancia con el objetivo de proporcionar a los padres y madres los recursos necesarios para fomentar una parentalidad positiva.

En lo que respecta a su efectividad, consideran queel castigo corporal solo tiene beneficios a corto plazo, en el momento de hacer desaparecer la conducta no deseada en el niño, pero no tiene una relación causal con el aprendizaje de conductas prosociales a largo plazo. De hecho, la mayor parte de la literatura asocia este tipo de violencia con desórdenes sociales y comportamientos agresivos futuros en el niño (Grogan-Kaylor, 2004; Straus, 1991; Straus y Yodanis, 1996), aunque en otros casos se demuestra que no todos los niños que han sufrido castigo corporal son violentos en la edad adulta (Bradley, 2001; Gershoff, 2002).

Algunos de los argumentos de los trabajadores sociales contrastan con la necesidad expresada de eliminar la aceptación social y cultural de la violencia. $Y$, aunque de sus palabras pudiera deducirse la importancia de sancionar comportamientos no deseados, es decir, la prohibición total del uso del castigo corporal, defienden que una reforma legislativa, por sí sola, no implica un cambio en las actitudes sociales. Conclusión a la que llegó también Roberts (2000) tras su investigación sobre la opinión de la población sueca tras veinte años desde la aprobación de la ley. Por lo tanto, toda acción legal debe acompañarse de intervenciones desde los servicios sociales, que asegure a las familias un soporte y una asistencia para el buen desarrollo de sus responsabilidades y competencias parentales.

Los resultados obtenidos son significativos por su imprevisión, al tratarse de un colectivo profesional en contacto diario con la infancia y al que se le atribuye un mayor conocimiento y sensibilización. En cada uno de los grupos de discusión se han generado opiniones no independientes sino relacionadas con el contexto en el que estaban siendo emitidas, pudiendo no coincidir sus manifestaciones verbales con sus actitudes o pensamientos reales. Los sentimientos se entremezclan, tal y como sucede en la realidad social, de tal manera que se puede afirmar que si "...el grupo reproduce el orden social existente" (Suárez, 2005: 32), la sociedad desconoce qué es el castigo corporal, aceptándolo desde la creencia de que no es una práctica negativa sino necesaria para conseguir una buena educación. Datos coincidentes con otros estudios (Horno, 2005) que señalan que la población española sigue tolerando ciertos grados de violencia contra los niños, estando mediatizada por una normas culturales; esto es, lo que la sociedad espera de la responsabilidad parental.

*Estudio financiado parcialmente en la Convocatoria de Proyectos de Investigación 2011-2012 del Departamento de Trabajo Social y Servicios Sociales de la Universidad Complutense de Madrid. 


\section{REFERENCIAS}

Bardin, L. (1986). El análisis de contenido. Madrid: Akal.

Belsky, J. (1993). Etiology of child maltreatment: a developmentalecological analysis. Psychological Bulletin, 114 (3), 413-434. doi:10.1037/0033-2909.114.3.413.

Berlin, L. J. y otros (2009). Correlates and consequences of spanking and verbal punishment for low income white, african american and mexican american toddlers. Child Development, 80 (5), 1403-1420. doi: 10.1111/j.14678624.2009.01341.x.

Bradley, R. H. (2001). The home environments of children in the United States part II: relations with behavioral development through age thirteen. Child Development, 72 (6), 1868-1886. doi: 10.1111/1467-8624.t01-1-00382.

Bussmann, K. D. ; Erthal, C. y Schroth, A. (2009). The effect of banning corporal punishment in Europe: A five-nation comparison. http://www.gruppocrc. net/IMG/pdf/Bussman_-_Europe_5_ nation_report_2009.pdf

Calvete, E.; Gámez-Guadix, M. y Orue, I. (2010). El inventario de dimensiones de disciplina (DDI), versión niños y adolescentes: Estudio de las prácticas de disciplina parental desde una perspectiva de género. Anales de Psicología, 26 (2), 410-418.

CIS: Centro de Investigaciones Sociológicas (2005). Actitudes y opiniones sobre la infancia. Estudio no 2621.

Comité de los Derechos del Niño (2006). Observación general no. 8: El derecho del niño contra la protección a los castigos corporales y otras formas de castigo crueles o degradantes (CRC/C/ GC/8). Génova, Suiza: ONU.
Comité de los Derechos del Niño (2011). Observación general no. 13: Derecho del niño a no ser objeto de ninguna forma de violencia. (CRC/C/GC/13). Génova, Suiza: ONU.

Del Río, P. y Álvarez, A. (1997). ¿Saber o comportarse? El desarrollo y la construcción de la directividad. En A. Álvarez (Eds.) Hacia un curriculum cultural. La vigencia de Vygotski en la educación (pp. 101-181). Madrid: Fundación Infancia y Aprendizaje.

Dietz, T. (2000). Disciplining children: Characteristics associated with the use of corporal punishment. Child Abuse and Neglect, 24 (12), 1529-1542. doi: 10.1016/S0145-2134(00)00213-1.

Durrant, J. (2003). Legal reform and attitudes toward physical punishment in Sweden. International Journal of Children's Rights. 11 (2), 147-173. doi: $10.1163 / 092755603322397241$.

Gershoff, E. T. (2002). Corporal punishment by parents and associated child behaviors and experiences: $A$ meta-analytic and theoretical review. Psychological Bulletin, 128 (4), 539-579. doi: $10.1037 / 0033-2909.128 .4 .539$.

Gershoff, E. T. y Bitensky, S. H. (2007). "The case against corporal punishment of children. Converging evidence from social science research and International Human Rights Law and implications for U.S. Public Policy" Psychology, public policy and law, 13 (4), 231-272. doi: 10.1037/1076-8971.13.4.231.

Global initiative to end all corporal punishment of children (2016). Corporal punishment of children: summaries of prevalence and attitudinal research in the last 10 years. http://www. endcorporalpunishment.org/research/ prevalence-research/ 
Gracia, E. y Herrero, J. (2008). Beliefs in the necessity of corporal punishment of children and public perceptions of child physical abuse as a social problem. Child Abuse and Neglect, 32 (11), 1058-1062. doi: 10.1016/j.chiabu.2008.05.004.

Grogan-Kaylor, A. (2004) "The effect of corporal punishment on antisocial behavior in children" Social Work Research, 28(3):153-164. doi:10.1093/ swr/28.3.153.

Hart, S. N.; Lee, Y. \& Wernham, M. (2011). A new age for child protectiongeneral comment 13: Why it is important, how it was constructed and what it intends. Child abuse and neglect, 35 (12), 970-978. doi 10.1016/j. chiabu.2011.09.007.

Hoffman, M. L. (1983). Affective and cognitive processes in moral internalization. En Higgins, D. N. Ruble y W. W. Hartup (Eds.). Social cognition and social development (pp. 236-274). New York: Cambridge University Press.

Holden, G. W., Coleman, S. M., \& Schmidt, K. L. (1995). Why 3-year old children get spanked: Parent and child determinants as reported by collegeeducated mothers. Merrill-Palmer Quarterly, 41(4), 431-452.

Horno, P. (2005). Amor, poder y violencia. Un análisis comparativo de los patrones de castigo físico y humillante. Madrid: Save the children.

Jackson, S. y otros (1999). Predicting abuse-prone parental attitudes and discipline practices in a nationally representative simple. Child Abuse and Neglect, 23 (1), 15-29. doi:10.1016/ S0145-2134(98)00108-2.

Juste, Ma G.; Morales, J. M. y Costa, M. (1997). Actitudes de los españoles ante el castigo físico infantil. Madrid: Ministerio de Trabajo y Asuntos Sociales. Krippendorff, K. (1990). Metodología de análisis de contenido. Teoría y práctica. Barcelona: Paidós Comunicación.
Lansford, J. E. y otros (2010). Corporal punishment of children in nine countries as a function of child gender and parent gender. International Journal of Pediatrics. doi: 10.1155/2010/672780.

Llopis, R. (2004). Grupos de discusión. Madrid: Esic Editorial.

MacKenzie, M. y otros (2011). Who spanks infants and toddlers? Evidence from the fragile families and child well-being study. Children and Youth Services Review, 33 (8), 1364-1373. doi: 10.1016/j.childyouth.2011.04.007.

Mahmoud, R., Salah-El-Din, M.y Ibrahim, M. (1998). Children experiencing violence I: parental use of corporal punishment. Child Abuse and Neglect, 22(10), 959-973. doi:10.1016/S01452134(98)00077-5.

Muller, R. T.; Hunter, J. E. y Stollak, G. (1995). The intergenerational transmission of corporal punishment: A comparison of social learning and temperament models. Child Abuse and Neglect, 19 (11), 1323-1335. doi:10.1016/0145-2134(95)00103-F.

Murillo, S. y Mena, L. (2006). Detectives y camaleones: el grupo de discusión. Madrid: Talasa Ediciones.

Picornell-Lucas, A. y otros (2014). La dignidad del menor en el entorno familiar y la facultad de corrección de los progenitores. En C. Lasarte (Dir.). Relaciones paterno-filiales, vol II, 115126. Madrid: Tecnos.

Pinheiro, P (2006). Informe para el estudio de la violencia contra los niños. Ginebra: ONU. http://www.unicef.org/ violencestudy/spanish/reports.html

Roberts, J. V. (2000): Changing public attitudes towards corporal punishment: the effects of statutory reform in Sweden. Child Abuse and Neglect, 24 (8), 1027-1035. doi: $10.1016 /$ S01452134(00)00155-1. 
Russell, A. y Russell, G. (1996): Positive parenting and boys' and girls' misbehaviour during a home observation. International Journal of Behavioral Development, 19 (2), 291-307. doi: $10.1080 / 016502596385794$.

Schmid, H. y Benbenishty, R. (2011). Public attitudes toward child maltreatment in Israel: Implications for policy. Child and Youth Services Review, 33, 1181-1188. doi: 10.1016/j. childyouth.2011.02.015.

Simons, D. y Wurtele, S. (2010): Relationships between parents use of corporal punishment and their children's endorsement of spanking and hitting other children. Child Abuse and Neglect, 34 (9), 639-646. doi: 10.1016/j. chiabu.2010.01.012.

Straus, M. A. (1991): Discipline and deviance: Physical punishment of children and violence and other crime in adulthood. Social Problems, 38, 133154. doi: $10.2307 / 800524$.

Straus, M. A. (2010). Prevalence, societal causes, and trends in corporal punishment by parents in world perspective. Law and contemporary problems, 73 (2), 1-30.

Straus, M. A. (ed.) (1994). Beating the devil out of them: Corporal punishment in American families. New York. Lexington Books.

Straus, M. A. y Fauchier, A. (2011). Manual for the dimensions of discipline inventory (DDI). http://pubpages.unh. edu/ mas2/DDI.htm

Straus, M. A. y otros (1998). Identification of child maltreatment with the parent-child conflict tactics scales: Development and psychometric data for a national sample of american parents. Child Abuse \& Neglect, 22 (4), 249-270. doi: 10.1016/S0145-2134(97)00174-9.
Straus, M. A., y Yodanis, C. L. (1996). Corporal punishment in adolescence and physical assaults on spouses later in life: What accounts for the link? Journal of Marriage and Family, 58(4), 825-841. doi: 10.2307/353973.

Suárez, M. (2005): El grupo de discusión. Barcelona. Laertes.

Tang, C. S. (2006). Corporal punishment and physical maltreatment against children: a community study on chinese parents in Hong Kong. Child Abuse \& Neglect, 30 (8), 893-907. doi: 10.1016/j.chiabu.2006.02.012.

Taylor, C. A. y otros (2010): Mother's spanking of 3 year old children and subsequent risk of children's aggressive behavior. Pediatrics, 125 (5), 10571065. doi: 10.1542/peds.2009-2678.

UNICEF (2003). A league table of child maltreatment deaths in rich nations: Innocenti Report Card No 5. Florencia: Innocenti Research Center. http:// www.unicef-icdc.org/publications/pdf/ repcard5e.pdf

Wareham, J.; Paquette, D. y Chavez, J. (2009): A test of social learning and intergenerational transmission among batterers. Journal of Criminal Justice, 37 (2), 163-173. doi: 10.1016/j. jcrimjus.2009.02.011.

Whipple, E. y Richey, C. (1997). Crossing the line from physical discipline to child abuse: How much is too much? Child Abuse and Neglect, 21(5), 431-444. doi: 10.1016/S0145-2134(97)00004-5.

Wolfe, D. A. (1985). Child abusive parents: an empirical review and analysis. Psychological Bulletin, 97 (3), 462-482. doi:10.1037/00332909.97.3.462. 